\title{
Neutrons magnetic Mass
}

manfred geilhaupt ( $\nabla$ manfred.geilhaupt@hsnr.de)

Hochschule Niederrhein https://orcid.org/0000-0001-8256-5627

\section{Research Article}

Keywords: Partiale physics, Neutron, Neutrino, beta-decay

Posted Date: December 13th, 2021

DOI: https://doi.org/10.21203/rs.3.rs-971432/v7

License: (c) (i) This work is licensed under a Creative Commons Attribution 4.0 International License. Read Full License 


\title{
Neutrons magnetic Mass
}

\begin{abstract}
:
In Quantum Physics, the Spin of an elementary particle is defined to be an intrinsic, inherent property. The same to the magnetic moment $(\mu)$ due to the spin of charged particles - like Electron $\left(m_{e}\right)$ and Proton $\left(m_{p}\right)$. So the intrinsic spin $(S=1 / 2 h-b a r)$ of the electron entails a magnetic moment because of charge (e). However, a magnetic moment of a charged particle can also be generated by a circular motion (due to spin) of an electric charge (e), forming a current. Hence the orbital motion (of charge around a massnucleus) generates a magnetic moment by Ampère's law. This concept must lead to an alternative way calculating the neutrino mass $\left(m_{v}\right)$ while looking at the beta decay of a neutron into fragments: proton, electron, neutrino and corresponding kinetic energies. The change of neutrons magnetic moment $\left(\mu_{n}\right)$ during the decay process is a fact based on energy and spin and charge conservation, so should allow to calculate the restmass of the charge-less neutrino due to a significant change of: $\mu_{\mathrm{e}}=-9.2847647043(28) \mathrm{E}-24 \mathrm{~J} / \mathrm{T}$ down to $\mu_{\mathrm{ev}}=-9.2847592533(28) \mathrm{E}-24 \mathrm{~J} / \mathrm{T}$ (while assuming $\mathrm{m}_{\mathrm{v}}=0.30 \mathrm{eV}$ to be absorbed and if (g-2)/2 from QED remains constant). As always the last word has the experiment.
\end{abstract}

\section{Introduction}

In short (using codata 2020): The fact that the charged particle $\left(m_{p}\right)$ is lighter than the „neutral“ one $\left(m_{n}\right)$ about $\left(m_{n}-m_{p}=1,29333235989 \mathrm{MeV}\right.$ or $m_{n}-m_{p}-m_{e}=0.78233341 \mathrm{MeV}$ or $\mathrm{mn} / \mathrm{mp}=1.00137841931$ ) remained mysterious up to now. The difference between the neutron mass $\left(m_{n}\right)$ and proton $\left(m_{p}\right)$ mass is an open question in Standard Model Physics [1]. Fact is, the neutron contains the mass of a neutrino (KATRIN $<1.10 \mathrm{eV}(2019)<0.80 \mathrm{eV}$ (2021) sensitivity $0.20 \mathrm{eV}$ ).

As shown by Cottingham [1], the magnetic mass is determined by the spin averaged forward Compton scattering amplitude. However, the Cottingham formula [1] did not explain the size of the observed mass differences with such a high accuracy ( $\left.\mathrm{m}_{\mathrm{QED}}=0,7(3) \mathrm{MeV}\right)[1]$ needed to come up with neutrino restmass estimation.

In this paper we start with the magnetic moment definition based on Ampère's law. From Ampère's law pure restmass without charge can not generate a magnetic moment. Thus the neutron mass must have an internal dynamic charge-action from a positive and negative parts (a least from quarks [1]) to come up with a magnetic moment and direction.

Elementary particles like electron, electron-neutrino and fundamental particles like proton, and neutron all obey the wave-particle behaviour as nature's fundamental fact, shown by Compton-Einstein [2]:

1. $h \cdot c / \lambda=m \cdot c^{2}$

Here Compton introduced the wavelength $(\lambda=c / f)$ instead of frequency ( $f$ ) as Einstein did concerning photo-electric effect. The Compton wavelength [2] $(\lambda)$ is a quantum mechanical wave-like value of a mass $(m)$ not zero (1.1). 


\section{$1.1 \lambda=h /(m \cdot c)$}

„The wavelength $(\lambda)$ was introduced by Arthur Compton in his explanation of the scattering of photons by electrons (Compton scattering)" [3] (velocity of light (c), Planck constant (h), based on Einsteins $E=m^{\star} c^{2}$ ).

Remark:

Compton-Einstein: $\quad$ wave $(\lambda)$ of a particle-mass $(\mathrm{m})$

Compton-Einstein: $\quad$ wave $(\lambda)$ of a particle-radius ( $\left.\mathrm{r}_{\mathrm{GN}}\right)$ [4]

\section{$1.2 \lambda=4 \pi \cdot r_{G N}$}

Let us (instead of $\lambda$ ) introduce an equivalent particle like parameter rGN [4] here by hypothesis, only to switch from the wave picture into a particle picture. Assuming the particle ist not point-like (and quantised due to N) [4] this allows to apply Ampère's Law $\left(I^{*} \Pi^{\star} r G N^{2}\right)$ in units $A^{*} m^{2}$.

Hint: $\mathrm{G}$ indicates a General Relativity theoretical fundament concerning the derivation of mass from theory [4] and $\mathrm{N}_{\mathrm{N}}$ formally indicates an intrinsic Quantum Number which respects the quantisation of mass of elementary and fundamental particles from a GR point of view. [4]

The intrinsic spin $\left(S=1 / 2^{\star} \hbar\right)$ due to $\mathrm{rGN}_{\mathrm{G}}$-interpretation can now be written being an invariant equation because of $S=1 / 2 h$-bar:

$$
1.3 S=c \cdot m \cdot r_{G N}
$$

If $m$ increases from electron to proton thus $\mathrm{rGN}_{\mathrm{G}}$ must decrease respectively while spin S remains invariant.

\section{Magnetic Moments and Ampère's Law}

In atomic physics, the Bohr magneton [5] $\mu_{\mathrm{B}}=\mathrm{e}^{*} \mathrm{~h} /\left(4 \pi^{*} \mathrm{~m}\right)$ in $\left[\mathrm{As}{ }^{*} \mathrm{Js} / \mathrm{kg}\right]$ is a „natural unit“ expressing the magnetic moment in Si-units [J/T=Am²] with $\left(T=1 \mathrm{~kg} / \mathrm{s} /\left(A^{*} \mathrm{~s}\right)\right.$ ) caused by either its spin-orbital or spin-angular momentum of a particle and mass $(\mathrm{m})$. The Magnetic Moment $(\mu)$ [5] while using Ampère's law [5] and 1.3 then reads $[3,4,5,6]$ :

$$
1.4 \mu=1 / 2 g_{s} \cdot e \cdot c \cdot r_{G N}
$$

in units $\left[A^{*} m^{2}\right]=[\mathrm{J} / \mathrm{T}]$

The Intrinsic Magnetic Moment [5] Dirac equation: $\mu=1 / 2 g_{s} \cdot e \cdot(1 / m) \cdot S$ depends on the Landé Factor gs, the charge $(\mathrm{e}=1.602176634 \mathrm{E}-19 \mathrm{C})$, velocity of light $(\mathrm{c}=2.99792458 \mathrm{E} 8 \mathrm{~m} / \mathrm{s})$, Planck Constant $(\mathrm{h}=6.62607015 \mathrm{E}-34 \mathrm{Js})$ and instead of particle parameter ( $(\mathrm{GN})$ or Compton wavelength $(\lambda)$ we use the mass $(\mathrm{m})$ as Dirac did. Thus 1.3 and 1.4 combined gives formula $\mu$ above. Now related to the particle radius $r_{G N}$. 


\section{Important to mention:}

Formulae 1.3 and 1.4 show an important energy based effect: If in (1.3) the mass $(m)$ increases (energy increases) so ( $\left.r_{G N}\right)$ decreases because the spin remains invariant. If in (1.4) ( $\left.r_{G N}\right)$ decreases then the magnetic moment $(\mu)$ decreases because the energy changed.

\section{Data from 2020}

We „use" $r_{G}$ instead of $r_{G N}$ now.

Of course the magnetic moment should be based on the quark model [1] on the level of the constituent quarks [6]. Yet our „simplified“ discussion is helpful to apply todays high accuracy Codata values.

\section{Free Proton:}

Magnetic moment: $\boldsymbol{\mu}_{\mathrm{p}}(+\mathbf{1 . 4 1 0 6 0 6 7 9 7 3 6 ( 6 0 ) E - 2 6 J / T )}$

$\mathrm{g}_{\mathrm{sp}}(5.5856946893(16))$ defined relative to the proton mass

Restmass: $m_{p}$ (1.67262192369(51)E-27kg)

- S-radius from (1.3): $\quad \mathbf{r g p}_{\mathrm{gp}}(\mathrm{S})=(1.051544551 \underline{67 \mathrm{E}}-16 \mathrm{~m})$

- $\mu$-radius from (1.4): $\quad$ rGp $(M)=(1.05154455167 \mathrm{E}-16 \mathrm{~m})$

\section{Free Neutron:}

Magnetic moment: $\boldsymbol{\mu}_{\mathbf{n}}(-9.6623651(23) \mathrm{E}-27 \mathrm{~J} / \mathrm{T})$

$g_{\text {snp }}(3.82608545(90))$ defined relative to the proton (not neutron) mass

Restmass: $m_{n}(1.67492749804 \mathrm{E}-27 \mathrm{~kg})$

- S-radius from (1.3): $r_{G n}(S)=1.05009707759 E-16 m$

- $\mu$-radius (1.4): $\quad r_{G n p}(M)=1.05154455167$ eE-16m

\section{Free Electron}

Magnetic moment: $\boldsymbol{\mu}_{\mathrm{e}}(-9.2847647043(28) \mathrm{E}-24 \mathrm{~J} / \mathrm{T})$

gse (2.00231930436256(35)) defined relative to the electron mass

Restmass: $m_{e}(9.1093837015(28) E-31 \mathrm{~kg})$

- S-radius from (1.3): $\mathrm{r}_{\mathrm{Ge}}(\mathrm{S})=1.9307963398 \mathrm{E}-13 \mathrm{~m}$

- $\mu$-radius from (1.4): $r \mathrm{Ge}(\mathrm{M})=1.9307963398 \mathrm{E}-13 \mathrm{~m}$ 


\section{Why a „mismatch“ for the neutron due to Codata's gsnp?}

The electron and proton using 1.4 and 1.3 go confirm (table 1). The neutron if we use $g_{s n p}=3.82608545(90)$ from Codata do not match. This $g_{s n p}$ here is related to the proton mass and not to the neutron mass as it should be if we want to apply 1.3 and 1.4. For that discussion we have to re-define $g_{s n}=g_{s n p}{ }^{*} m_{n} / m_{p}=3.831359 \underline{40}$. Then 1.4 works for the neutron assuming a "dominating“ negative charge (although compensated by a positive charge) defining the direction of the magnetic Moment.

Overview: (2019-Codata $(\mathrm{m}),(\mu),(\mathrm{g})$, from $1.3 \mathrm{rGx}_{\mathrm{G}}(\mathrm{S})$, from $\left.1.4 \mathrm{rGx}(\mathrm{M})\right)$

\begin{tabular}{|c|c|c|c|c|}
\hline $\begin{array}{l}\text { Codata } \\
2019\end{array}$ & $\begin{array}{l}\text { Restmass (m) } \\
\text { In kg }\end{array}$ & $\begin{array}{l}\text { Magnetic Moment ( } \mu \text { ) } \\
\text { In J/T }\end{array}$ & Landé Factor (g) & $\begin{array}{l}r G x(S) \text { in } m \\
r G x(M) \text { in } m\end{array}$ \\
\hline $\begin{array}{l}\text { Electron } \\
\mathrm{mn} / \mathrm{me}\end{array}$ & $\begin{array}{l}9.1093837015(28) \mathrm{E}-31 \\
1838.6836617\end{array}$ & $-9.28476470 \underline{43}(28) E-24$ & $\begin{array}{l}2.002319304362 \underline{56} \\
\text { (35) }\end{array}$ & $\begin{array}{l}1.9307963398 \mathrm{E}-13 \\
1.9307963398 \mathrm{E}-13\end{array}$ \\
\hline Muon & $1.8835316 \underline{27}(42) \mathrm{E}-28$ & $-4.490448 \underline{30}(10) \mathrm{E}-26$ & $2.00233184 \underline{18(13)}$ & $\begin{array}{l}9.3379715299 \mathrm{E}-16 \\
9.3379715376 \mathrm{E}-16\end{array}$ \\
\hline Tauon & $3.16754 \mathrm{E}-27$ & No value & No value & $5.5526890611 \mathrm{E}-17$ \\
\hline $\begin{array}{l}\text { Proton } \\
\mathrm{mp} / \mathrm{me}\end{array}$ & $\begin{array}{l}1.672621923 \underline{69}(51) \mathrm{E}-27 \\
1836.15267 \underline{\underline{34}}\end{array}$ & $1.410606797 \underline{36}(60) \mathrm{E}-26$ & $5.58569468 \underline{93}(16)$ & $\begin{array}{l}1.0515445517 \mathrm{E}-16 \\
1.0515445517 \mathrm{E}-16\end{array}$ \\
\hline $\begin{array}{l}\text { Neutron } \\
\mathrm{mn} / \mathrm{mp}\end{array}$ & $\begin{array}{l}1.67492749804(95) \mathrm{E}-27 \\
1.001378419 \underline{13}\end{array}$ & $-9.66236 \underline{1}(23) \mathrm{E}-27$ & $3.831359 \underline{40}$ & $\begin{array}{l}1.0500970776 \mathrm{E}-16 \\
1.0515445517 \mathrm{E}-16\end{array}$ \\
\hline
\end{tabular}

Table 1 Magnetic Moments and „magnetic“ Mass equivalent $\left(E=m c^{2}\right), x=(e, p, n)$

Magnetic Moments and Neutrons Mass from Codata (2020).

The (magnetic) mass of the Neutron (including $\mathrm{m}_{\mathrm{v}}$ neutrino) is that of the experimental mass $m_{n}=1.67492749804 \mathrm{E}-27 \mathrm{~kg}$. The (magnetic) mass of the proton $\mathrm{m}_{\mathrm{p}}=1.672621923 \underline{69} \mathrm{E}-27 \mathrm{~kg}$ not including the neutrino mass.

From (1.3) $\mathrm{r}_{\mathrm{n}}(\mathrm{S})=1.05009707 \underline{76} \mathrm{E}-16 \mathrm{~m}$ while using the neutron restmass $\left(\mathrm{m}_{\mathrm{n}}\right)$.

From (1.4) the magnetic moment $\mu_{n}\left(g_{n p}\right)=-9.6623651(23) E-27 \mathrm{~J} / T$ from experiment now based on the proton mass $\left(m_{p}\right)$, and $r_{n n}(M)=1.0500970748 \mathrm{E}-16 \mathrm{~m}$ from $\mu_{n}\left(g_{n}\right)=-9.6623651(23) E-27 \mathrm{~J} / T$ here based on the Neutron mass $\left(m_{n}\right)$ must be combined with $\mathrm{g}_{\mathrm{n}}=3.83135940$.

From (1.4) the same magnetic moment from experiment of the neutron due to $\mathrm{g}_{\mathrm{np}}$ $\mu_{n}\left(g_{n p}\right)=-9.6623651(23) E-27 J / T$ while here $g_{n p}$ is based on $m_{p}$ (proton mass), gives:

$r_{G n p}(M)=1.0515445516 \mathrm{E}-16 \mathrm{~m}$ form $\mathrm{mM}$ corresponding to the proton mass $\mathrm{m}_{\mathrm{p}}$.

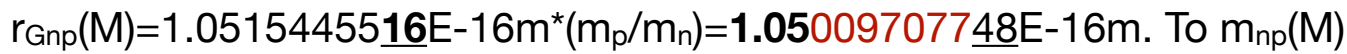

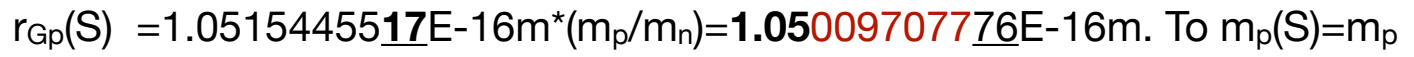

From $1.4 m_{n p}(M)=938272088.23 e V$ (including the Neutrino $m_{v}$ ) and $m_{n p}(M)-m_{p}=S / c^{\star}\left\{1 / r_{G n p}(M)-\left(1 / r_{G p}(S)\right\}=0.099 e V\right.$ (not acceptable accuracy)

\section{Remark on absolute accuracy limits}

$g_{n p}=3.82608545(90)$ and $\mu_{n}=-9.6623651(23) E-27 \mathrm{~J} / T$ error-limits giving the maximum absolut error-bars (+444.15 and - 443.95)eV while calculating the corresponding mass values from 1.4. From this we get the „mean relative error" (0.20)eV „estimation“ while assuming gaussian shaped statistics. 


\section{Conclusion:}

Binding Energy from Codata restmass values:

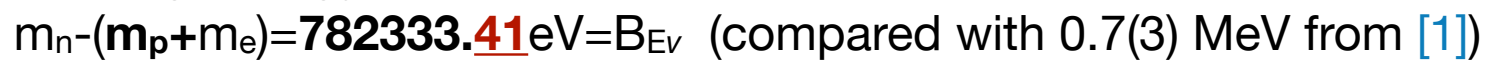

$\mathrm{B}_{\mathrm{Ev}}=\mathrm{B}_{\mathrm{E}}+\mathrm{m}_{\mathrm{v}}$, including" anti-neutrino restmass reads:

$B_{E}=m_{n}-\left(m_{p}+m_{e}+m_{v}\right)=782333$.??eV after decay

(Katrin $<1.1 \mathrm{eV}$ Cosmological observations suggest that the mass of neutrinos could be $0.17 \mathrm{eV}$ or lighter. [8])

However, the neutrino restmass estimation $(0.10(20) \mathrm{eV}$ might be possible.

But accuracy limits from Codata 2019 are excluding any such speculation at the moment. Nevertheless speculation beyond SM is possible.

\section{Beyond the SM:}

The Spin (S), charge, velocity of light and energy all must be invariant during the process. Mass $(\mathrm{m})$, radius $\left(\mathrm{r}_{\mathrm{G}}\right)$, and magnetic moment $(\mu)$ must not be constant. This is true for electron and proton and neutron mass steps.

$1.3 S=c \cdot m \cdot r_{G N}$

$1.4 \mu=1 / 2 g_{s} \cdot e \cdot c \cdot r_{G N}$

Notice: $\mathrm{N}$ formally respects quantisation of radius or mass $\mathrm{m}(\mathrm{N})$ respectively [4].

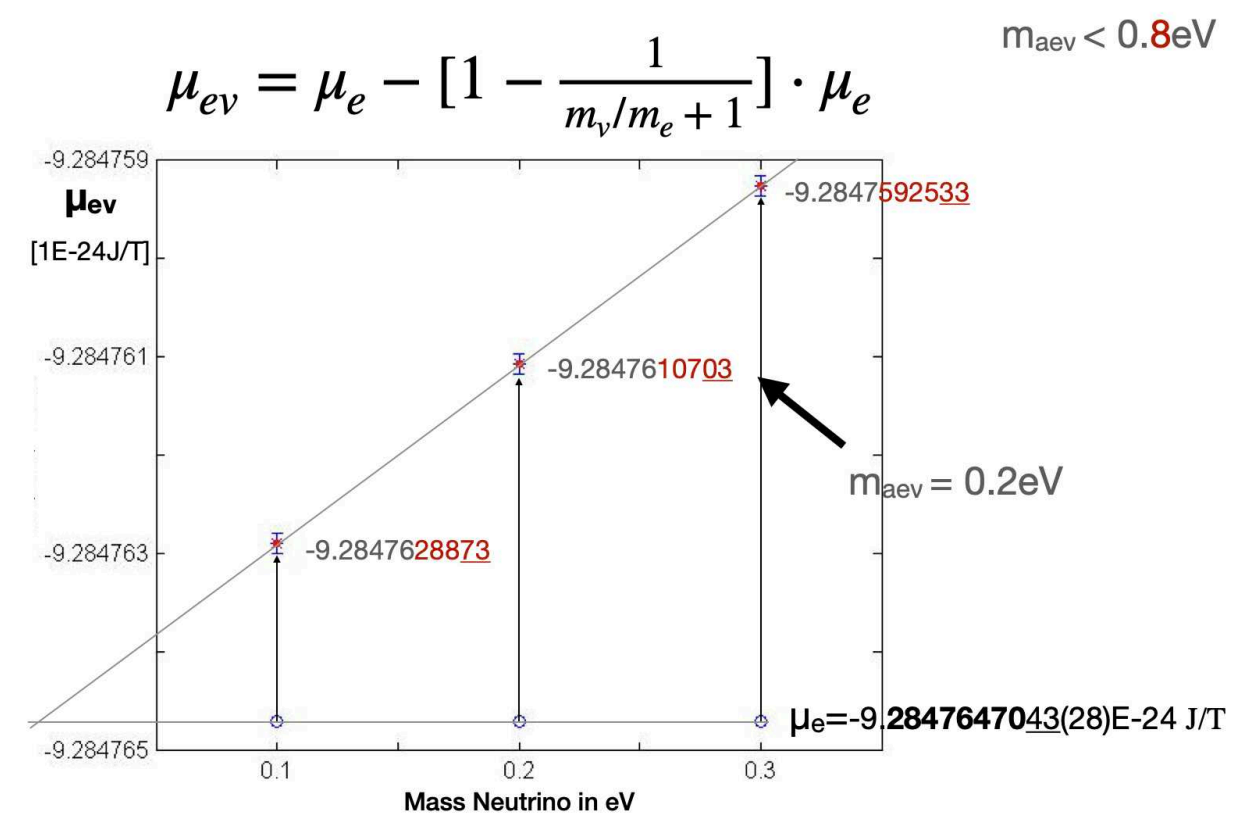

Figure 1 Hypothesis: If the electron absorbed a neutrino (after beta-decay) the magnetic moment changes significantly, as shown for $0.1 \mathrm{eV}, 0.2 \mathrm{eV}$, and $0.3 \mathrm{eV}$ compared with the experimental value (or $0.0 \mathrm{eV}$ ) due to high accuracy experiment possible to measure. 
Remark magnetic moment change:

Within the beta-decay process, while releasing binding energy, the neutron (change its magnetic moment), so releases a proton (its mass and its magnetic moment and its kin. energy) and at the same time releases an electron (its mass and its magnetic moment and its kin. energy).

Suppose electrons are aloud to keep an anti-neutrino „inside“ (absorbed) after the decay process (me+mv $>$ me). So the magnetic moment of those electrons (still absorbing an electron-neutrino) is smaller.

Notice:

getheory $=2.00231930436322(46)$ the SM prediction (from Wiki 2020) compared to the experimental number $\mathrm{g}_{\mathrm{e}}=2.00231930436256$ (35) (from Wiki 2019 ) is matching $100 \%$. The discrepancy is maximum $0.38^{*} 10^{-6} \mathrm{eV}$ and minimum $0.06^{\star} 10^{-6} \mathrm{eV}$ estimated from the error limits of $g_{\mathrm{et}}$ and $\mathrm{ge}_{\mathrm{e}}$.

$$
1.5 d m_{e}=\left(g_{e t} / g_{e}\right) \cdot m_{e}-m_{e}
$$

The calculation ( $d_{m e}$ ) shows: QED prediction and experimental value are in full agreement. So here no electron-neutrino was involved. We have to look for electron keeping absorbed an electron-neutrino! And what happens with get for that too? (Both effects would not compensate each other.) 


\section{Literature}

(1) J. Gasser, H. Leutwyler, A. Rusetsky On the mass difference between proton and neutron Physics Letters B Volume 814, 2021. (https://www.sciencedirect.com/science/article/pii/S0370269321000277?via\%3Dihub)

(2) The Dirac Electron: Spin, Zitterbewegung, the Compton Wavelength, and the Kinetic Foundation of Rest Mass, Herausgeber: Kiyoshi Nishikawa, Jean Maruani, Erkki J. Brändas, Print ISBN: 978-94-007-5296-2 or ISBN: 978-94-007-5297-9Gerardo Delgado-Barrio, Piotr Piecuch, Verlag: Springer Netherlands

(3) Compton scattering: https://en.wikipedia.org/wiki/Compton wavelength

(4) Derivation of Radius (Particle restmass ( $\mathrm{rGN}$ ) behaves like a wave!) https://www.researchsquare.com/article/rs-524770/v3

Only if a GR-theory is able to derive $r_{G N}$ exclusively. Thus we will have a theoretical answer for the open question: "Why does the Compton Wavelength exist from a theoretical point of view already proved by Compton scattering experiment?"

(5) Magneton https://en.wikipedia.org/wiki/Bohr_magneton Intrinsic Magnetic Moment https://de.wikipedia.org/wiki/Land\%C3\%A9-Faktor

(6) Constituent quarks and magnetic moment https://de.wikipedia.org/wiki/Proton Große Deutsche Enzyklopädie „Damit ein Elementarteilchen ein intrinsisches magnetisches Moment hat, muss es sowohl Spin als auch elektrische Ladung haben. Das Neutron hat einen Spin von $1 / 2 \hbar$, aber keine Nettoladung. Die Existenz des magnetischen Moments des Neutrons war rätselhaft und widersetzte sich einer korrekten Erklärung, bis in den 1960er Jahren das Quarkmodell für Partikel entwickelt wurde. Das Neutron besteht aus drei Quarks, und die magnetischen Momente dieser Elementarteilchen verbinden sich, um dem Neutron sein magnetisches Moment zu verleihen. “

(7) More accuracy Double-trap measurement of the proton magnetic moment at 0.3 parts per billion precision Schneider et al., Science 358, 1081-1084 (2017)

(8) https://www.nature.com/articles/d41586-019-02786-z 